\title{
Leading-Proton Production in DIS at HERA
}

\author{
L. Rinaldi, on behalf of the ZEUS Collaboration
}

INFN Bologna

\begin{abstract}
The semi-inclusive reaction $e^{+} p \rightarrow e^{+} X p$, with a final-state proton carrying a large fraction of the incoming proton energy, $x_{L}>0.5$, and transverse momentum squared $p_{T}^{2}<0.5 \mathrm{GeV}^{2}$, was studied with the ZEUS detector at HERA for exchanged photon virtualities $Q^{2}>3 \mathrm{GeV}^{2}$ and masses of the photon-proton system $45<W<225 \mathrm{GeV}$, using an integrated luminosity of $12.8 \mathrm{pb}^{-1}$. Leading protons were detected with the ZEUS leading-proton spectrometer. The leading-proton production cross section and rates are presented as a function of $x_{L}, p_{T}^{2}, Q^{2}$ and the Bjorken scaling variable, $x$.
\end{abstract}

\section{Introduction}

Events with a final-state proton carrying a large fraction of the available energy, $x_{L}$, but a small transverse momentum, $p_{T}$, have been studied in detail in high-energy hadron-proton collisions $[2,3,4,5,6]$. More recently, the HERA-collider experiments reported measurements of the production of leading protons in $e p$ collisions $[7,8]$. Several mechanisms have been suggested to explain the production of leading protons (LP). None of them are, as yet, amenable to calculations based on perturbative quantum chromodynamics (pQCD). This is, in part, a consequence of the small values of $p_{T}$ of the leading proton which necessitates a non-perturbative approach. Some models [9] are based on the Regge formalism, with leading-proton production occurring through $t$-channel exchanges, both isoscalar and isovector, notably of the Pomerons, Reggeons and pions. These exchanges mediate the interaction between the proton and the hadronic fluctuations of the virtual

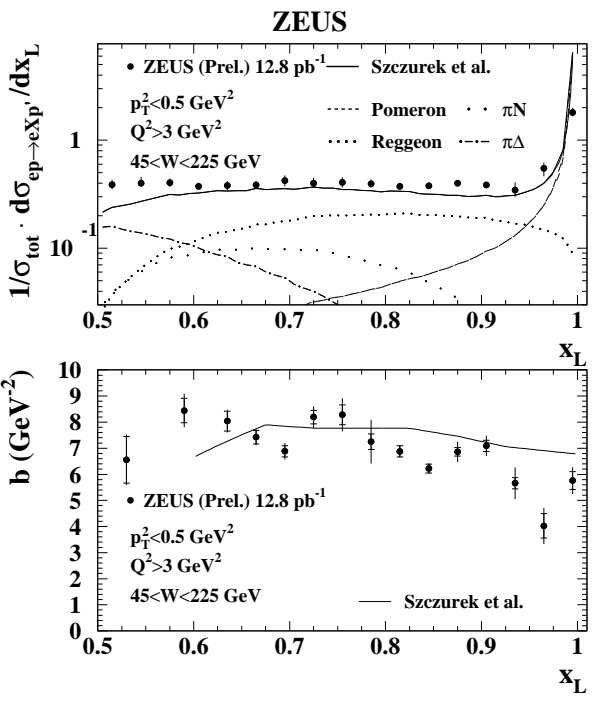

Figure 1: A Regge-based model [9] compared to the normalized cross section, $1 / \sigma_{t o t}$. $d \sigma_{\mathrm{LP}} / d x_{L}$, and $p_{T}^{2}$-slope, $b$.

photon. Other models retain quarks and gluons as fundamental entities, but add nonperturbative elements, such as soft-colour interactions (SCI) [10].

\section{Experimental Setup, Data Selection and Monte Carlo Simulation}

The measurement was performed with data collected in 1997 at the ep collider HERA using the ZEUS detector. A detailed description of the ZEUS detector can be found elsewhere [11].

The component most relevant for this study was the leading-proton spectrometer (LPS) [12]. It was used during the HERA I data taking period (1994-2000) to detect positively charged 
particles scattered at very small angles and carrying a large fraction of the longitudinal momentum of the incoming proton. It consisted of 54 planes of silicon microstrip detectors grouped into six stations, S1 to S6, and located along the outgoing proton beam line between $Z=20 \mathrm{~m}$ and $Z=90 \mathrm{~m}$.

The amount of data recorded when the experimental conditions allowed the LPS to be operational correspond to an integrated luminosity of $12.8 \mathrm{pb}^{-1}$. Two sets of events were selected [13], the inclusive DIS sample and the LPS sample, both in the kinematic domain $Q^{2}>3 \mathrm{GeV}^{2}$ and $45<W<225 \mathrm{GeV}$.

For the DIS sample the following cuts were required: $\left|Z_{\mathrm{vtx}}\right|<50 \mathrm{~cm}$, where $Z_{\mathrm{vtx}}$ is the $Z$ coordinate of the event vertex; energy of the scattered positron $E_{e}^{\prime}>10 \mathrm{GeV}$; the quantity $E-P_{Z}$, where the energy $E$ and the longitudinal momentum $P_{Z}$ are summed over all the energy deposits and the scat-

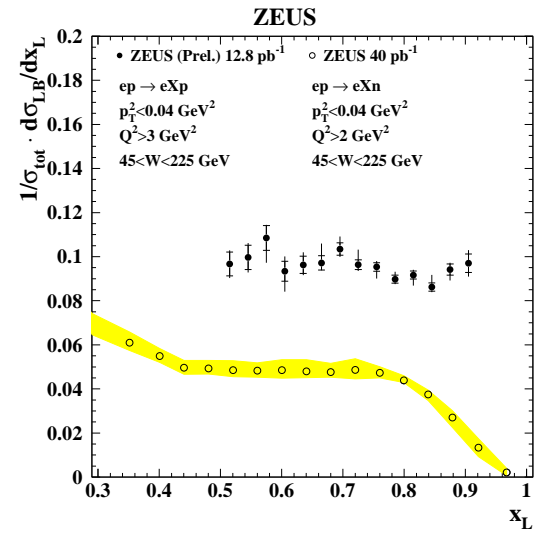

Figure 2: Production rate, $1 / \sigma_{t o t}$. $d \sigma_{\mathrm{LB}} / d x_{L}$, for leading-proton and leading-neutron production. tered positron, in the range $38<E-P_{Z}<65 \mathrm{GeV} ; y_{\text {JB }}>0.03$ in order to ensure hadronic activity away from the forward direction.

The LPS sample was obtained by selecting events that satisfy the inclusive DIS selection as above, requiring in addition a LPS dedicated trigger and a reconstructed track in the LPS with $p_{T}^{2}<0.5 \mathrm{GeV}^{2}$ and $x_{L}>0.5$. To reduce the sensitivity of the LPS acceptance due to the uncertainties of the location of the beampipe elements, a cut was applied to the minimum distance, $\Delta_{\text {pipe }}$, between the track and the beampipe requiring $\Delta_{\text {pipe }}>0.04 \mathrm{~cm}$. The selection of tracks with $\Delta_{\text {pot }}>0.02 \mathrm{~cm}$, where $\Delta_{\text {pot }}$ is the minimum distance of the track from the edge of any LPS detector, ensured that the tracks were well within the active regions of the silicon detectors. The sum of the energy and the longitudinal momentum of both the energy deposits in the CAL and the particle detected in the LPS, $E+P_{Z}$, was required to be smaller than $1655 \mathrm{GeV}$; this cut rejected most of the random overlays of DIS events with protons from the beam-halo or from a proton-gas collision.

To determine the acceptance of the apparatus, inclusive DIS events with $Q^{2}>0.5 \mathrm{GeV}^{2}$ were generated using DJANGOH [14]. In order to study the migration of events from low $Q^{2}$, a sample of photoproduction events with $Q^{2}<0.5 \mathrm{GeV}^{2}$ was generated with PYthia [15]. The diffractive events in DJANGOH were generated using the soft-colour-interaction mechanism [10].

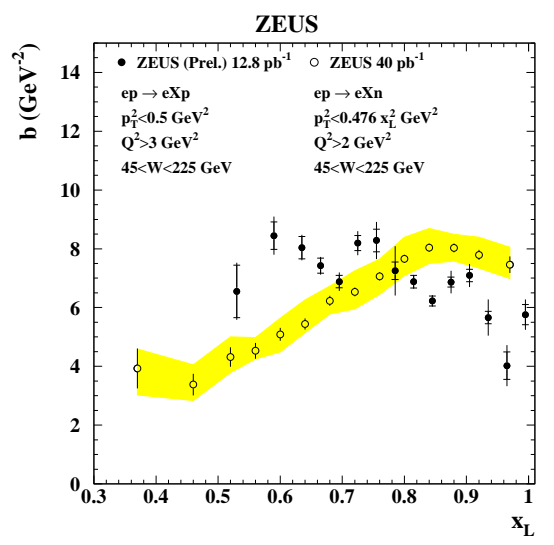

Figure 3: The $p_{T}^{2}$-slope, $b$, as a function of $x_{L}$ for leading-proton and leading-neutron production.

All the MC events were passed through the standard simulation of the ZEUS detector and trigger and through the same reconstruction and analysis programs as the data.

To obtain a good description of the data, it was necessary to reweight the leading-proton 
$x_{L}$ and $p_{T}^{2}$ distributions generated by the MC [13].

The systematic uncertainties were calculated by varying the cuts and by modifying the analysis procedure. The main contribution is given by the variation of the reweighting parameters, the simulated transverse momentum spread of the proton beam and the positions of the LPS stations in the MC simulation.

\section{Results}

The normalized cross section, $1 / \sigma_{\text {tot }}$. $d \sigma_{\mathrm{LP}} / d x_{L}$, where $s i g m a_{\text {tot }}$ was determined from the inclusive DIS sample, was measured for $p_{T}^{2}<0.04 \mathrm{GeV}^{2}$ and $p_{T}^{2}<0.5$ $\mathrm{GeV}^{2}$. The $p_{T}^{2}$-slope, $b$, was obtained by a fit of an exponential function $A \cdot e^{-b p_{T}^{2}}$ to the cross section $d^{2} \sigma_{\mathrm{LP}} / d x_{L} d p_{T}^{2}$, measured in bins of $x_{L}$.

Figure 1 shows the predictions of the model of Szczurek et al. [9] are compared to the leading-proton production rate $1 / \sigma_{\text {tot }}$. $d \sigma_{\mathrm{LP}} / d x_{L}$ and the $p_{T}^{2}$-slopes. In this model,

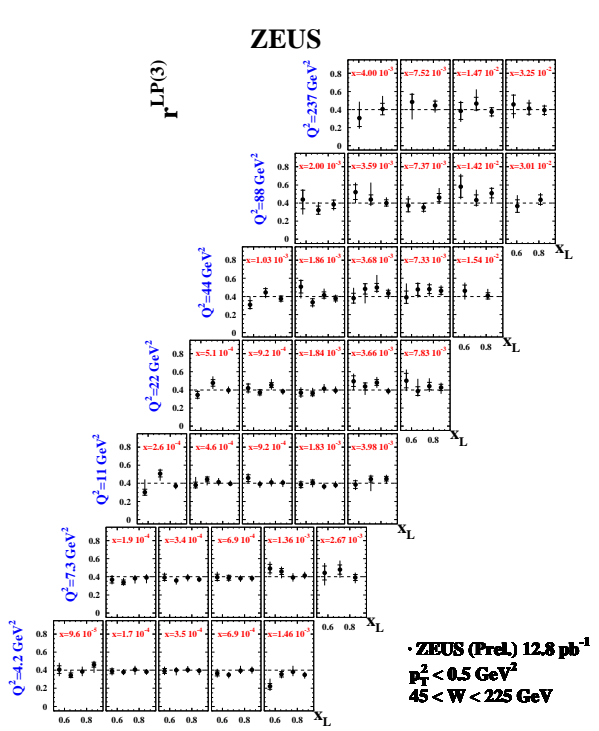

Figure 4: The LP production rate $r^{\mathrm{LP}(3)}$ as a function of $x_{L}$ in bins of $x$ and $Q^{2}$. leading-proton production for $0.6<x_{L}<0.9$ is dominated by isoscalar Reggeon exchange. Diffractive processes due to Pomeron exchange become increasingly important as $x_{L}$ approaches unity. The contribution of pion exchange plays an important role in the medium $x_{L}$ range. The model describes reasonably well the shape of the longitudinal momentum spectrum and of the $p_{T}^{2}$-slopes.

The rate of leading-proton production as a function of $x_{L}$ was compared to the recent measurement of leading neutrons [16] for $p_{T}^{2}<0.04 \mathrm{GeV}^{2}$. The comparison is shown in Fig. 2. In the range $0.5<x_{L}<0.92$, there are approximately twice as many protons as neutrons. In a particle exchange model, the exchange of isovector particles would result in half as many protons as neutrons. Thus, exchange of isoscalars must be invoked to account for the observed proton rate [9]. This contribution is likely also to explain the different behavior of the rates at large $x_{L}$.

The slopes of the $p_{T}^{2}$ distributions for leading protons and neutrons are shown in Fig. 3. Although the $p_{T}^{2}$ and $Q^{2}$ ranges are different, the two samples have similar values of $b$ in the region $0.65<x_{L}<0.8$, where pion exchange is expected to dominate the production mechanism [9].

The rate of leading-proton production, $r^{\mathrm{LP}(3)}\left(x, Q^{2}, x_{L}\right)$, in $e^{+} p$ scattering was determined as $r^{\mathrm{LP}(3)}\left(x, Q^{2}, x_{L}\right)=A \frac{N^{\mathrm{LPS}}\left(x, Q^{2}, x_{L}\right)}{N^{\mathrm{DIS}}\left(x, Q^{2}\right)} \frac{1}{\Delta x_{L}}$, where $N^{\mathrm{LPS}}\left(x, Q^{2}, x_{L}\right)$ is the number of events with a proton candidate in the LPS in a given $x, Q^{2}, x_{L}$ bin and $N^{\mathrm{DIS}}\left(x, Q^{2}\right)$ is the number of DIS events in that $x, Q^{2}$ bin, the variable $\Delta x_{L}$ indicates the size of the $x_{L}$ bin and the variable $A$ includes the acceptance and the selection efficiency. 
The ratio $r^{\mathrm{LP}(3)}$ as a function of $x_{L}$ in bins of $x$ and $Q^{2}$ is shown in Fig. 4. The $r^{\mathrm{LP}(3)}$ values are approximately constant over the kinematic range of this analysis.

The rate of leading-proton production as a function of $x$ and $Q^{2}$, integrated over $0.5<x_{L}<0.92$ and $p_{T}^{2}<0.5 \mathrm{GeV}^{2}$, $r^{\mathrm{LP}(2)}\left(x, Q^{2}\right)$, is shown in Fig. 5. The ratio $r^{\mathrm{LP}(2)}$ is approximately constant as a function of $x$ and of $Q^{2}$. The mean value $\left\langle r^{\mathrm{LP}(2)}\right\rangle \approx 0.17$ means that approximately $17 \%$ of inclusive DIS events with $p_{T}^{2}<0.5$ $\mathrm{GeV}^{2}$ have a leading proton in the range $0.5<x_{L}<0.92$.

The ratios $r^{\mathrm{LP}(3)}$ and $r^{\mathrm{LP}(2)}$ were used to extract the leading-proton structure functions $F_{2}^{\mathrm{LP}(3)}\left(x, Q^{2}, x_{L}\right)$ and $F_{2}^{\mathrm{LP}(2}\left(x, Q^{2}\right)$, (more details and plots in [1]). The LP structure functions are proportional to the proton structure function $F_{2}$.

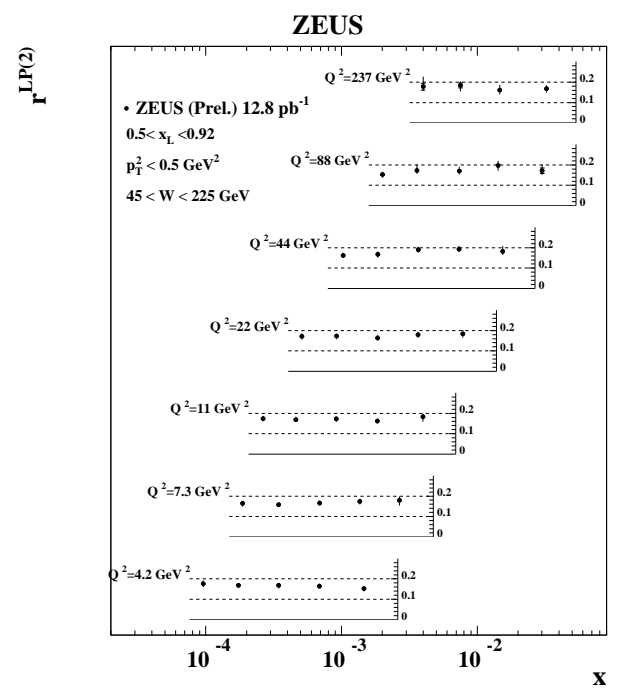

Figure 5: The LP production rate $r^{\mathrm{LP}(2)}$ as a function of $x$ in bins of $Q^{2}$.

\section{References}

[1] Slides: http: //indico. cern. ch/contributionDisplay py? contribId=25\&sessionId=16\&conf Id=24657

[2] V.N. Gribov et al., The creation of $Q C D$ and the effective energy, L.N. Lipatov (ed.), World Scientific Series in 20th Century Physics, Vol. 25, World Scientific, Singapore (2001).

[3] M. Basile et al., Nuovo Cimento 66A, 129 (1981); M. Basile et al., Lettere al Nuovo Cimento 32, 321 (1981).

[4] J. Whitmore and M. Derrick, Phys. Lett. B 50, 280 (1974).

[5] A. E. Brenner et al., Phys. Rev. D 26, 1497 (1982).

[6] M. Aguilar-Benitez et al., Z. Phys. C 50, 405 (1991).

[7] ZEUS Collab., S. Chekanov et al., Nucl. Phys. B 685, 3 (2003).

[8] H1 Collab., C. Adloff et al., Eur. Phys. J. C 6, 587 (1999); H1 Collab., C. Adloff et al., Nucl. Phys. B 619, 3 (2001).

[9] A. Szczurek, N.N. Nikolaev and J. Speth, Phys. Lett. B 428, 383 (1998).

[10] A. Edin, G. Ingelman and J. Rathsman, Phys. Lett. B 366, 371 (1996).

[11] ZEUS Collab., U. Holm (ed.), The ZEUS Detector, Status Report (unpublished) DESY, 1993, available on http://www-zeus.desy.de/bluebook/bluebook.html.

[12] ZEUS Collab., M. Derrick et al., Z. Phys. C 73, 253 (1997).

[13] L. Rinaldi, Ph.D. thesis, University of Bologna (2006), Report DESY-THESIS-2006-028.

[14] G. A. Schüler and H. Spiesberger, Proceedings of the Workshop on Physics at HERA, Volume 3, W. Buchmüller and G. Ingelman (eds.), DESY (1991), p. 1419;

H. Spiesberger, DJANGOH, http://www.desy.de/ ^hspiesb/djangoh.html.

[15] T. Sjöstrand et al., Comp. Phys. Comm. 135, 238 (2001).

[16] ZEUS Collab., S. Chekanov et al., Nucl. Phys. 776, 1 (2007). 